\title{
Designing a Serious Game for Community-Based Disease Prevention in the Amazon
}

\author{
Saturnino Luz \\ School of Comp. Sci. and Stat. \\ Trinity College Dublin \\ luzs@cs.tcd.ie
}

\author{
Masood Masoodian \\ Dept. of Computer Science \\ The University of Waikato \\ masood@waikato.ac.nz
}

\author{
Manuel Cesario \\ Acad. AIAOASSCA+CECS \\ Franca-SP, Brazil \\ manuel.cesario@uol.com.br
}

\author{
Raquel Rangel Cesario \\ Medical School \\ University of Franca \\ raquelrangel-ac@uol.com.br
}

\author{
Bill Rogers \\ Dept. of Computer Science \\ The University of Waikato \\ coms0108@waikato.ac.nz
}

\begin{abstract}
Many developing regions around the world rely on community-based healthcare strategies and practices to deal with prevention and control of often neglected diseases, by educating the local population and healthcare professionals, on the mechanisms by which such diseases spread and how they can be controlled. In this paper we describe a multiplayer serious game designed to raise awareness, and foster adoption of preventive measures among local citizens and community-health professionals about Leishmaniosis. We also discuss how the underlying concept for this game and its mechanics have been iteratively designed and developed in collaboration with a group of people with relevant medical and research expertise as well as practical knowledge resulting from working with our target population.
\end{abstract}

\section{Author Keywords}

Serious games; games for healthcare; community education; disease prevention; mobile computing.

\section{ACM Classification Keywords}

H.5.m. Information Interfaces and Presentation (e.g. HCI): Miscellaneous

\section{INTRODUCTION}

One of the aims of community-health work is to help citizens and community healthcare professionals better understand the complex mechanisms involved in human and animal parasitic disease transmission, and encourage the adoption of prevention strategies. This need is particularly important in developing countries, where the considerable economic and social impact of the so called "neglected diseases" [2] could be minimized through effective education campaigns. This is specifically the case of Visceral Leishmaniasis (VL), a disease whose incidence rate has increased in recent years.
The research reported in this paper investigates the potential usefulness of a serious game as an educational tool in the prevention of VL. This work is underpinned by the OneHealth theoretical framework [13], which approaches healthcare as a multidisciplinary effort encompassing all aspects of care for humans, animals and the environment. VL is an ideal case for application of OneHealth concepts because it involves not only humans, dogs and insect vectors (phlebotomine sandflies), but also silvatic reservoirs (e.g. foxes) and domestic animals (e.g. chickens) that attract both vectors and silvatic reservoirs to the peri-domicile, composing a complex and poorly-understood transmission cycle.

The work described here forms part of a strategy to break the VL's transmission chain in communities, through an online serious game incorporating the OneHealth paradigm within an interactive educational tool. We introduce Dr Ludens' $L S G$ (Leishmaniasis Serious Game), which is designed to involve local citizens and community-health professionals in cooperative tasks around their households and communal places, where they will aim to decrease vector density and to protect people and dogs against VLs transmission.

\section{SERIOUS GAMES}

The importance of play in human culture is first discussed in Huizinga's Homo Ludens [3]. He points out that play is fun and free, creates order, and develops knowledge; and that while doing and daring are power, knowing is magical power! Play also allows people to safely explore the consequences of their actions, or inaction. Furthermore, as Koster [5] argues, the fundamental pleasure people gain from playing games is the satisfaction of learning.

It is therefore natural that educators have been keen to leverage the widespread popularity of computer games to what is basically a "learning" activity in a more directed way. However, despite some notable successes such as Math Blasters (see [10]) which offers students shoot-em-up rewards for maths drill, and SimCity which gives students control over the simulated economic and physical development of a city, experience with educational computer games in many cases has not been successful [11]. Reasons for this failure include the tendency to be overly explicit about learning objectives and to include conventional assessment activities [11]. 
More recent work in serious games and gamification offer new ideas for embedding games into real-life situations, making use of people's interest in gaming to directly achieve useful results, and using social interaction to provide motivation. For instance, Cooper et al. [1] showed how a protein folding game could use the efforts of a large number of online players to solve a complex computational problem.

Applications of serious games in the healthcare are also becoming widespread. In a recent survey, Wattanasoontorn et al. [12] identified over a hundred different games focusing on personal health issues, encouraging people to learn to manage their own health problems, motivating rehabilitation, and so on. Although games in the public health area are less common, there are a few examples. For instance, the Public Health Preparedness Island [9] uses an archipelago of islands with a range of urban and rural settings to provide a simulation to help train people to deal with a variety of emergency scenarios, such as pandemic influenza, bio-terrorism, etc.

\section{MULTIDISCIPLINARY GAME DESIGN TEAM}

Design and development of Dr Ludens' LSG by our team has relied on close collaboration between the game designers and a group of practitioners and researchers working in areas related to the topic of the game. This multidisciplinary research group includes medical doctors, veterinarians, entomologists, epidemiological surveillance professionals, public health professionals, and social scientists who for the last decade have been working on various OneHealth issues in connection with Leishmaniases surveillance at the deprived tri-national borders of the South-Western Amazonia.

The activities of this group include: (a) identification of the main factors involved with the hyperendemicity of American Cutaneous Leishmaniasis (ACL) in Acre-Brazil through a ten-year retrospective multivariate statistical analysis; (b) use of qualitative field-based ethnographic work to validate transmission factors in the Brazilian municipality of Assis BrasilAcre, at the Madre de Peru-Brazil-Bolivia borders; (c) ongoing research on the regional migration patterns (international and national, in Brazil and Peru) and public policies related to the (re)emergence of Leishmaniases; (d) comprehensive ongoing research aimed at developing an Early Warning System for Bartonellosis and Leishmaniases at the tri-national region, drawing from the OneHealth paradigm and including Climate Change variables; (e) ongoing development of a computer-based tele-health system for handheld mobile devices, including applications for epidemiological surveillance data-sharing, GIS visualization of disease cases, and remote diagnosis support for Bartonellosis and Leishmaniases [7].

\section{A SERIOUS GAME FOR LEISHMANIASES}

The idea of employing a serious game is an unconventional approach to disease control, considering the fact that the existing efforts to control VL transmission have relied on pharmaceutical and biomedical developments, since the identification of the antimonials for treatment. The development of chemicals either to break disease transmission with the appropriate treatment of humans and, in some countries, dogs, or to prevent people and dogs getting infected by the use of insect repellents, and the development of vaccines (for dogs, so far) have helped at a slower pace than the spread of the disease. The World Health Organization lists all types of Leishmaniases as neglected diseases.

Our working assumption is that when Dr Ludens' LSG is made available to local people living in the targeted region, they are not only likely to play the game and get excited about it, but will also learn from the information embedded in the game and change their behaviours in relation to preventive measures for Leishmaniases.

The basis for this assumption is that the use of computers and computer-games is well-spread in this region, even among the lower income individuals living in remote places. We have observed that in places like the Brazilian municipality of Assis Brasil-Acre and the Peruvian locality of Iñapari-Madre de Dios, on opposite sides of the South-Western Amazonia borders, where internet connectivity is still poor, local people, especially teenagers (who tend to have lower incomes), either gather with their own portable computers in the very few places with Wi-Fi connection, or go to some sort of internetcafe when they do not have their own computers, to interact with others and play online games.

In addition to local people, Dr Ludens' LSG will also target healthcare professionals and scientists involved in the prevention and epidemiological study of VL, by providing a scientifically accurate simulation model. As pointed out in a recent global health foresight report, "hybridizing such games with robust simulation could help health workers and scientists visualize how diseases and interventions might play out" [8].

\section{GAME CONCEPT}

Dr Ludens' LSG has been designed to be played on a range of devices (mobile phones, tablets, and PCs) connected asynchronously to a server. This is to allow people with access to different types of devices, and various levels of internet connectivity to play the game.

Players may choose between a rural and an urban visual environment. After exploring the chosen setting, they will identify Dr Ludens (the in-game expert doctor) and engage in a quiz-like discussion with him, in which they will acquire the initial information relating to the tasks they need to perform. The initial tasks to be performed by players include: identifying the sandfly vectors of Leishmaniases among different insects; identifying main breeding sites for sandflies in their chosen settings; removing organic matter (litter inadequately disposed, as well as leaves, branches, and dirt water) around their houses and/or in communal places. The performance of those tasks for a given period of time will enable the player to acquire and store points, and to move to the next game level.

At the higher levels, more complex tasks will be required by Dr Ludens, such as: placing appropriate nets on doors and windows to decrease the number of sandflies inside the house; acquiring, installing in an appropriate way, and using bed-nets every night; moving domestic animals that ordinarily live freely around the house to appropriate enclosures away from the house, to act as a buffer against the approximation of the sandflies from the surrounding forests or bushes. 
These more complex tasks may be performed individually to collect points, but will gain extra points if the player engages other players to work with them, fostering cooperation. On the other hand, if the player fails to demonstrate that, for instance, the nets are being used appropriately, or the animals are being kept in an enclosure away from the house, they will loose some of their stored points; thus risking to return to a lower level of the game.

As the game progresses, Dr Ludens will provide more sophisticated quizzes and discussions, and incorporate correspondingly more complex tasks, such as the adequate use of insect repellent lotions for people and collars for dogs. At the highest levels, Dr Ludens will provide state-of-the-art scientific information for the players, always using easy to understand language. He will also promote discussion on keytopics related to OneHealth aspects of VL prevention, such as: schemes for people's treatment; people's vaccination (or not); vaccination and/or treatment of dogs (or not); up to the highly sensitive determination (in Brazil) of euthanasia for dogs with Leishmaniasis.

\section{SOFTWARE ARCHITECTURE}

The basic software architecture of the game consists of the following components, as laid out on Figure 1:

- A simulator (Badagua), whose function is to simulate human, animal and epidemiological activity within a geographical region using sophisticated simulation models.

- A game client (DrLudensLSG), which allows players to interact with the game using a range of devices.

- A game server (Dr Ludens' Gatekeeper, or DLGatekeeper for short), whose function is to coordinate each player's access to their "household", record their interaction, scores, etc. and generally mediate the communication between Badagua and DrLudensLSG.

Badagua, the simulation backend, is a discrete, multi-agent simulator written in Java, using the Mason library [6]. The simulator represents the map of the region where the game takes place as a sparse 2D grid. This grid is subdivided into households, which are assigned to individual players. These subdivisions are permeable while the simulation is global, so that the state of one household may affect all households. For instance, if a player allows water to accumulate in their area

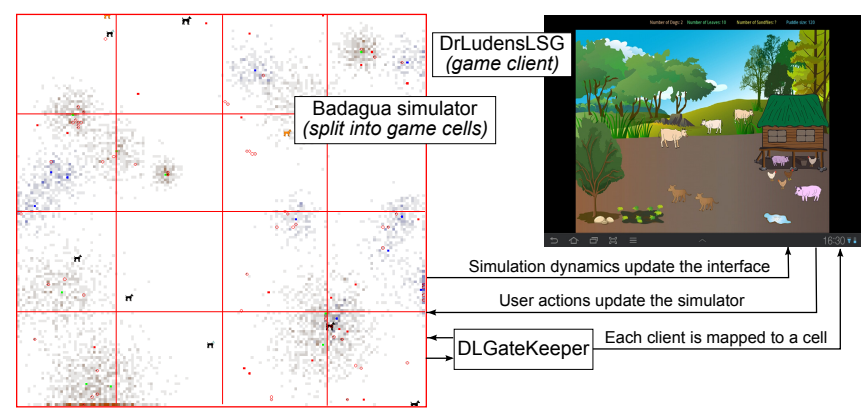

Figure 1. Software architecture of Dr Ludens' LSG, showing its three main components, and interactions between them. and this leads to sandfly infestation, this infestation might also cause members of other nearby households to become infected. An important aspect of the game is to raise awareness for these kinds of environmental effects among the players.

The map of the simulator is populated by agents that implement the dynamics of (1) water, leaf and refuse accumulation, (2) movements and infection state of domestic animals, and (3) sandfly reproduction, movements, and infection state. All these agents are parametrized, so that diseases and sandfly cycles can be modelled according to actual scientific knowledge and data. We use a simplified SEIR (Susceptible, Exposed, Infectious, Recovered) model to simulate disease spread [4].

The communication between Badagua and DrLudensLSG is bidirectional, with the user interface component prompting changes to the underlying simulation, and querying the simulator periodically so as to reflect changes brought about by its environmental dynamics.

\section{DESIGN AND DEVELOPMENT OF THE GAME CLIENT}

The design of the game client of Dr Ludens' LSG and the higher-level design of the overall game concept as described above, started with simple sketches, which formed the basis for the initial discussions between the game designers and the subject matter specialists and practitioners.

One of the sketches used for these discussions was that of a map, as shown in Figure 2, over which interactions between multiple game players residing in the region of interest were developed. This sketch presents the entire world of Dr Ludens' LSG simulated by Badagua, which contains individual households, each of which belongs to one of the players.

We also used various sketches of a farm (household) allocated to individual game players. The visual design of the farm was developed using some free vector graphics stock which we adopted and modified in consultation with our local experts to represent a typical village setting in the South-Western Amazon region.

Once the visual design of the main elements of the game client developed, we coded its interactive components. To make the game widely available, our local experts requested that the game client should be available on mobile devices, as

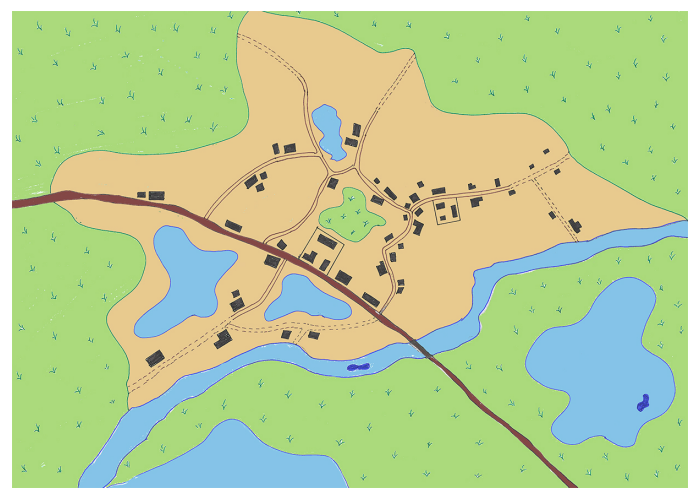

Figure 2. Sketch of the map of an area in which different households allocated to different players are represented. 


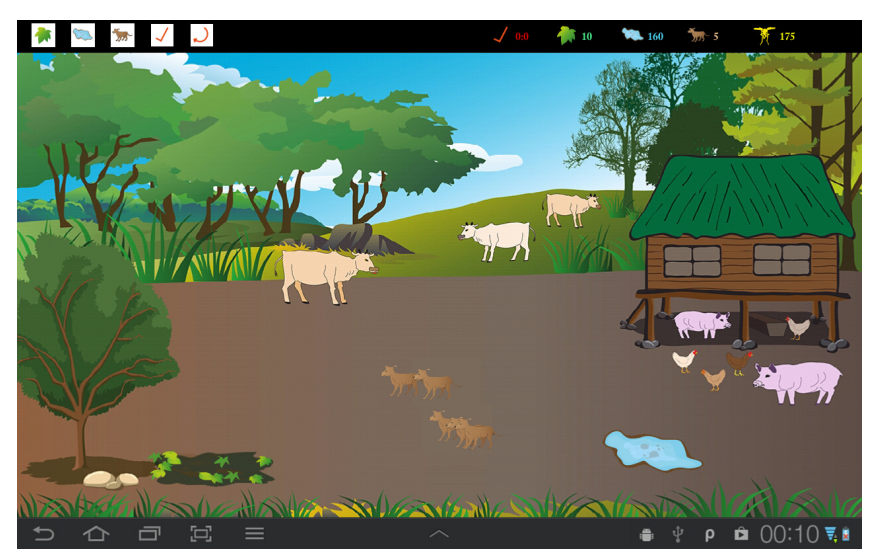

Figure 3. Dr Ludens' LSG game client on a Samsung ${ }^{t m}$ Tab 10.1.

well as standard PCs, both as a native application and through web browsers. We chose Processing for coding our game client prototype, as it supports development of standard Java, JavaScript, and Android applications.

Figure 3 shows the interface of the current implementation of the game client of Dr Ludens' LSG for Android-based mobile devices. The central part of the interface displays a typical village setting, with a hut and various animals, some of which are kept under the hut. The visual look of the game client and its basic layout are the same across different implementations for various devices.

As mentioned earlier, some of the player's objectives in the game are to remove infected stray dogs (Figure 4, left), clean leaves (Figure 4, centre), and dry water puddles (Figure 4, right) which all act as reservoirs and breeding areas for sandflies. These tasks can be achieved by selecting an appropriate tool, shown on the top left-hand side of the screen (see Figure 3), and then clicking on dogs, leaves or the water puddle. These will change the game score and statistics shown on the top right-hand side of the screen (see Figure 3).
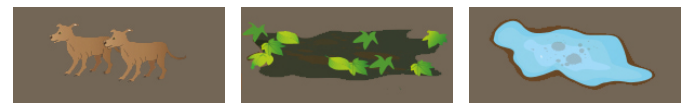

Figure 4. The player needs to destroy infected dogs (left), clean leaves (centre), and dry puddles (right) to reduce the number of sandflies.

Dr Ludens' LSG also uses quizzes to encourage learning information about Leishmaniases, its spread, methods of control, prevention, etc. Answering quizzes correctly allows the player to collect points which can then be used in the game.

\section{CONCLUSION AND FUTURE WORK}

In this paper we have discussed the potential benefits of the use of serious games as part of community-based healthcare practices, and presented Dr Ludens' LSG as one such game.

Dr Ludens' LSG has been designed in close collaboration with a group of experts involved in monitoring and control of Leishmaniases, and several other similar diseases, in trinational region of the Amazon. We have also recently made our game available to a selected group of initial users. After gathering sufficient feedback from this pilot group, we plan to make any required changes and enhancements and then release the game to the local population in our region of interest.

Once Dr Ludens' LSG has been made available to the public, we intend to conduct a longitudinal study involving collection and analysis of game play statistics, and a formal intervention study. These will guide future developments of Dr Ludens' LSG, and the design of similar games for other diseases.

\section{REFERENCES}

1. Cooper, S., Khatib, F., Treuille, A., Barbero, J., Lee, J., Beenen, M., Leaver-Fay, A., Baker, D., Popovic, Z., and players, F. Predicting protein structures with a multiplayer online game. Nature 466 (2010), 756-760.

2. Hotez, P. J., Fenwick, A., Savioli, L., and Molyneux, D. H. Rescuing the bottom billion through control of neglected tropical diseases. The Lancet 373, 9674 (2009), 1570-1575.

3. Huizinga, J. Homo ludens: A study of the play-element in culture. Beacon Press Boston, MA, 1967.

4. Keeling, M. J., and Rohani, P. Modeling infectious diseases in humans and animals. Princeton University Press, 2008.

5. Koster, R. Theory of Fun for Game Design, 2nd ed. O'Reilly Media, 2013.

6. Luke, S., Cioffi-Revilla, C., Panait, L., Sullivan, K., and Balan, G. Mason: A multiagent simulation environment. Simulation 81, 7 (2005), 517-527.

7. Luz, S., Masoodian, M., and Cesario, M. Disease surveillance and patient care in remote regions: an exploratory study of collaboration among health-care professionals in Amazonia. Behaviour \& Information Technology (2014).

8. Masum, H., Ranck, J., and Singer, P. A. Five promising methods for health foresight. Foresight 12, 1 (2010), 54-66.

9. Monahan, C., Ullberg, L., and Harvey, K. Virtual emergency preparedness planning using second life. In Proceedings of the IEEE/INFORMS International Conference on Service Operations, Logistics and Informatics, SOLI '09, IEEE (2009), 306-310.

10. Rice, J. Assessing higher order thinking in video games. Journal of Technology and Teacher Education 15, 1 (January 2007), 87-100.

11. Shelton, B. E., and Wiley, D. Instructional designers take all the fun out of games: Rethinking elements of engagement for designing instructional games. In Proceedings of the Annual Meeting of the American Educational Research Association (2006).

12. Wattanasoontorn, V., Boada, I., García, R., and Sbert, M. Serious games for health. Entertainment Computing 4, 4 (December 2013), 231-247.

13. Zinsstag, J., Schelling, E., Waltner-Toews, D., and Tanner, M. From "one medicine" to "one health" and systemic approaches to health and well-being. Preventive veterinary medicine 101, 3 (2011), 148-156. 